\title{
Additive Solution to Diastema Closure by a Combination of Direct and Indirect Techniques
}

\author{
Jesus Oteo* \\ Professor, Departamento Odontología Conservadora, Spain
}

Keywords: Diastema; Closure; Smile; Esthetic

\section{Introduction}

Smile design in restorative dentistry embraces facial esthetics, gingival esthetics, the relationship between soft and hard tissues and dental asthetics $[1,2]$. Smile design requires assessment of facial soft tissues and skeleton, followed by intraoral examination of the teeth and their relationship with lips and soft tissues [3]. A facial analysis is performed to determine how lips and soft tissue frame the smile in different positions of speech and laughter and establishes the localization of the facial midline and its position with respect to the dental midline. Smile parameters include: gingival marginal levels tooth size, including tooth-tooth proportion and tooth width: length ratio; tooth position; tooth shape; incisal embrasures; connector spaces, where adjacent teeth appear to touch; axial inclinations; and the shade of maxillary teeth [2].

Studies on the attractiveness of smiles as a function of their variation from esthetic norms have shown the presence of a diastemato be a decisive negative factor and a strong candidate for treatment $[4,5]$.

Adiastema is a black space between adjacent teeth that are separated from each other, with no presence of a contact area. Possible origins of this defect include an excessively wide dental arch, anomalous tooth size, congenital tooth absence and gingival frenum hypertrophy. First, it is important to differentiate diastemata from pathologic tooth migration pathologic tooth migration that have developed over time and may indicate a lack of stability in tooth position [6]. A decision must then be taken whether to treat the patient with a multidisciplinary approach or simply to close the spaces by means of direct and/or indirect restorative therapy [7].

A spaced dentition also can be due to various reasons such as hypodontia, tooth size discrepancy and impeded eruption. The dilemma for clinicians is whether to close, open or redistribute the space [8]. Two principles govern the prosthodontic management of dentitions that are compromised by missing teeth or by size or shape alterations: the first principle is to obtain appropriate spaces for the optimal replacement of missing teeth with teeth of suitable size or shape; and the second is for existing teeth to be optimally positioned within the dental arch [9]. The ideal space size for restoration in these cases is established by measuring the contralateral tooth; if this is present in correct proportions, Bolton tooth-size analysis (or similar) is performed, determining average width: height ratios of the teeth and preparing an orthodontic setup or diagnostic wax-up [9].

If the teeth are correctly positioned and anomalous tooth size is the origin, the clinician can choose between restoration with porcelain or composite resin, which have different properties and imply distinct clinical procedures [10]. A direct or indirect approach can be used with the latter, but the use of porcelain requires an indirect technique. The decision to use one or the other depends on the characteristics of each case. If the diastema results from tooth malposition, orthodontic treatment can avoid the need for a restorative approach [11]. When there is a congenital absence of teeth or the presence of gingival frenum hypertrophy, surgery may be required $[12,13]$.
The aim of this case report is to show how to solve a problem of black spaces preserving the major possible quantity of sound teeth by means of combining different materials with excellent esthetic results

\section{Case Report}

A healthy 27-year-old man presented to the clinic due to interdental spaces (Figure 1). He expressed satisfaction with the color of his teeth and a desire to avoid additional orthodontic treatment. His wide smile showed a high upper lip line. Incisal edges of anterior teeth were wellpositioned in relation to gingival margins. The patient had no carious lesions or history of restorative treatment. He had a thin gingival biotype and fairly symmetrical gingival architecture.

Temporomandibular joints were normal, with no history of dysfunction. Although the patient's visits to the dentist had been infrequent, clinical examination evidenced good periodontal health, with no probing depths $>3 \mathrm{~mm}$ and periapical radiography studies showed adequate bone levels. Microdontia of the upper incisors produced diastemata in the esthetic zone.

Among the different possible approaches to this case, we selected the most conservative because of the good periodontal status of the patient and his desire to improve his appearance.

Before the treatment, his medical and dental history was reviewed, preoperative radiographs and photographs were taken and periodontal charting was done. Diagnostic impressions were made and study models were mounted on an articulator. Smile analysis was performed by digitally tracing over a photograph (1:2 proportion). A diagnostic wax-up with modeling-wax (Eco-Cera Dental S. L. Monachil. Granada. Spain) of the proposed ideal was made for the creation of a mockup, using silicone key (Polixilosane Lab-Putty, ColteneWhaledent. Altstätten/Switzerland) (Figure 2) and filling it with Protemp 3 Garant

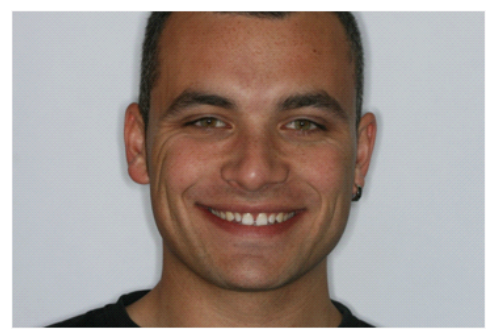

Figure 1: Patient's interdental spaces.

*Corresponding author: Jesus Oteo, Professor, Departamento Odontología Conservadora, Spain, E-mail: dentaloteo@telefonica.Net

Received March 01, 2012; Accepted March 09, 2012; Published March 11, 2012

Citation: Oteo J (2012) Additive Solution to Diastema Closure by a Combination of Direct and Indirect Techniques. Dentistry 2:125. doi:10.4172/2161-1122.1000125

Copyright: (c) 2012 Oteo J. This is an open-access article distributed under the terms of the Creative Commons Attribution License, which permits unrestricted use, distribution, and reproduction in any medium, provided the original author and source are credited. 
A3 shade (3M ESPE Dental Products. St. Paul, MN. USA). This model also served to create a putty index to enable the preservation of as much of the dental structure as possible in the preparation (Figure 3). Tooth shade evaluation of his natural teeth indicated the use of Vitapan Classical B1 shade (VITA Zahnfabrik. Bad Säckingen, Germany) with white horizontal lines.

In the preparation of the right lateral incisor, the silicon index was used to ensure adequate removal of tooth structure for the ceramic layering. The orientation of the teeth in the arch indicated a porcelain width of $1.2 \mathrm{~mm}$ at the facial aspect and $1.3 \mathrm{~mm}$ at the incisal edge. Because the cervical margin was subgingival, Ultrapak \#000 and \#00 retraction cords (Ultradent Products. South Jordan UT. USA) were packed to obtain a profile of gradual emergence that ended in a slight chamfer. A minimal vestibular reduction of $0.1 \mathrm{~mm}$ was needed to regularize the surface. No removal of dental tissue was necessary to produce a space in the incisal third of the tooth.

The left lateral incisor required a minimal preparation of around $0.5 \mathrm{~mm}$ in the mesial area of the buccal aspect and $0.3 \mathrm{~mm}$ in the distal area of the buccal aspect. An incisal preparation of $0.5 \mathrm{~mm}$ was sufficient to create internal characteristics in the porcelain that mimicked the natural tooth. The interproximal preparation was transferred in a lingual direction to prepare the interdental porcelain extension with a profile of progressive emergence in both lateral incisors (Figure 4). Free-hand composite resin enamel plus HFO (Micerium. Avegno. Italy) was used to create direct temporary restorations.

When the veneers were received from the laboratory, their fit was tested in the model, verifying the presence of adequate contact points with the adjacent teeth and performing an in-mouth color test with temporary Variolink Veneer try-In glycerin-based cements. (IvoclarVivadent. Schaan. Liechtenstein). Veneers were etched with $10 \%$ hydrofluoric acid (IvoclarVivadent. Schaan. Liechtenstein) for 90 seconds and the residue was then removed by immersion in an ultrasonic cleaner with $95 \%$ alcohol. Next, two coats of Silane (IvoclarVivadent. Schaan, Liechtenstein) were placed and then dried to evaporate the solvent. The veneers were placed in a black box during the dental preparation. The anterior area of the maxilla was isolated with an extra-thick (15 x $15 \mathrm{~cm})$ Hygenic dental dam, separating neighboring teeth with $12 \mathrm{~mm} \times 12 \mathrm{~mm} \times 0.075 \mathrm{mmPTFE}$ Teflon strip (Unimax International Limited. Cixi, Ningbo, P. R. China). The surface was etched with $37 \%$ orthophosphoric acid (Ultradent Products. South Jordan UT. USA) for 30 seconds and the teeth and veneers were prepared with Ena-Bond light curing bonding (Micerium, Avegno, Italy). The final cementation step was resin cementation with lightcuring adhesive luting compositeVariolink Veneer high value +1 (IvoclarVivadent. Schaan. Liechtenstein) on both surfaces removing remanent cement with paint brush and dental floss (Figure 5). All resin cement was removed from the subgingival area by using a scalpel blade. The occlusion was then re-tested, making any necessary corrections with a fine-grain diamond bur. Finally, the margins were polished with diamond discs.

For the two central incisors, a diamond bur was used to create a rough surface, removing enamel prisms and enhancing the adhesion. The right central incisor was restored first, polishing the interproximal aspect, followed by restoration and polishing of the left central incisor. The same steps were followed for both, etching with $37 \%$ phosphoric acid for 30 seconds (Ultradent Products, South Jordan UT, USA), rinsing clean and then lightly air-drying to confirm a proper etch pattern. Ena-Bond light curing bonding agent (Micerium, Avegno, Italy) was painted on the entire tooth surface, dried with an air syringe

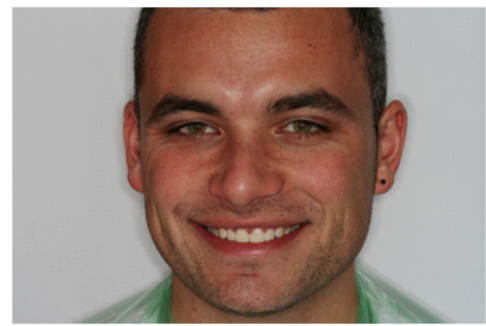

Figure 2: A Mock up of the proposed ideal

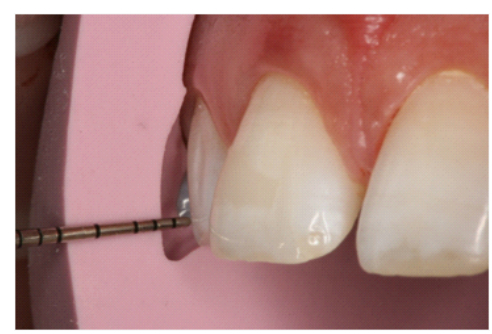

Figure 3: A putty index was created to preserve as sound dental structure as possible.

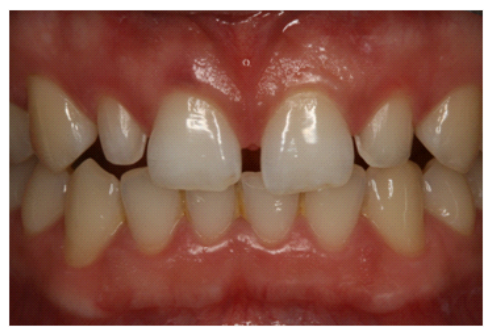

Figure 4: A minimal reduction was needed for lateral incisors.

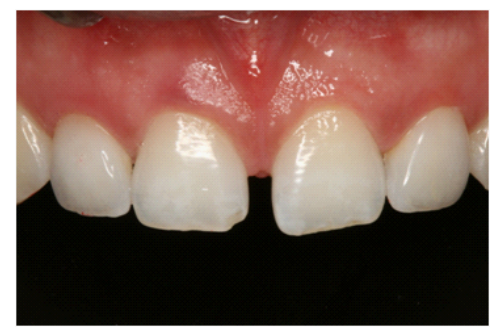

Figure 5: Veneers cemented on lateral incisors

until no movement was observed and then light-cured for 10 seconds. Composite layering followed, curing each layer for 10 seconds after its placement. A $0.3 \mathrm{~mm}$ palatal layer of Enamel plus HFO shade GE3 (Micerium, Avegno, Italy) was applied on the silicon key to conform the palatal aspect of the restoration. A small amount of Enamel plus dentin shade UD2 (Micerium, Avegno, Italy) was applied on interproximal surfaces. An Ambar translucent shade was applied on the incisal third to create an internal effect of translucency. A Kolor + plus Resin Color Modifier kit (Kerr Corporation. OrangeCA 9286. USA) was used to apply white stains to reproduce the natural stains observed in the tooth, followed by light-curing for 10 seconds. A fine layer of Enamel microfill GE3 (Micerium. Avegno. Italy) was placed to create the gross shape of 
the central incisors. This final shade was then covered with gel-oxalate to complete the polymerization.

After analyzing the photographic studies, the patient was seen for modification of the form and shape of the direct veneers. Final polishing with Sof-Lex burs and discs (3M ESPEDental Products. St. Paul, MN. USA) from larger to smaller grain was done to obtain a satin-like finish on facial composite surfaces. Secondary and tertiary anatomies were reproduced with a fine grain conical diamond burn. Composite polishing pastes Enamel plus shiny 3 and 1 micron diamond pastes and Enamel plus shiny $\mathrm{C} 1$ micron aluminum oxide (Micerium, Avegno, Italy) were used on Felt disc Shiny FD 5, (Micerium, Avegno, Italy) to achieve a high gloss finish (Figure 6). the patient's smile satisfied the patient's demands (Figure 7). 18-Month follow-up image depicts the harmonious integration of form and color achieved (Figure 8).

After treatment was a total integration of the restorations in the mouth of the patient. The new appearance of the patient's smile satisfied the patient's demands (Figure 7). 18-Month follow-up image depicts the harmonious integration of form and color achieved (Figure 8).

\section{Discussion}

The clinician primarily assessed this case from an esthetic viewpoint, achieving a natural outcome despite the use of different materials on adjacent teeth. Numerous authors have demonstrated that

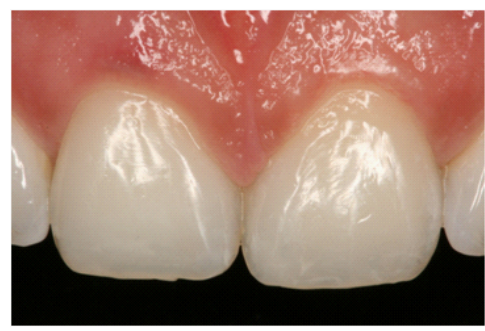

Figure 6: Definitive composite restorations after finishing and polishing procedures.

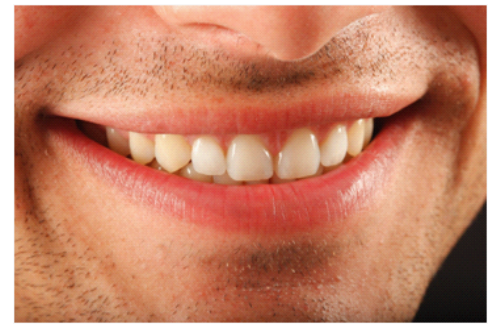

Figure 7: Integration of the restorations in the mouth of the patient.

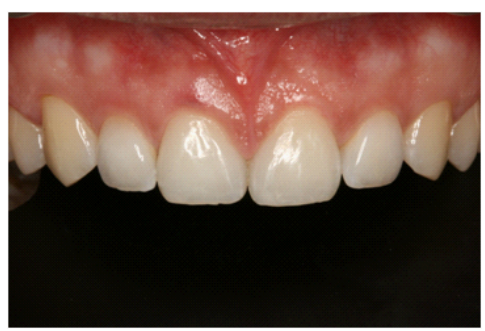

Figure 8: 18-month follow -up image depicts the harmonious integration of form and color achieved. diagnosis efforts and the study of patients' individual characteristics are key to a successful esthetic outcome $[14,15]$ and a diagnostic wax-up and corresponding template can be used to evaluate the result in the patient's mouth before beginning the treatment $[16,17]$.

The patient's perception of cosmetic improvement is not significantly affected by the selection of the material (direct composite resin or porcelain) for maxillary anterior veneers, although a more conservative option of composite veneers may be preferred by patients given the choice after receiving adequate information [18]. In the present case, the clinician offered the patient an additive solution for his teeth, adding cosmetic materials and avoiding reduction of his dental structure.

A silicon index of the additive wax-up is used as a reference for tooth reduction [19], allowing a minimal reduction and therefore the optimal outcome. In the present patient, the silicon index showed that more space was available for a restoration in the lateral incisors than in the central incisors.

Pascal Magne [20] described the importance of an extensive reduction in order to close diastemata, with an interproximal preparation in lingual and subgingival directions to prepare an interdental extension of the porcelain with a profile of progressive emergence. This entails an aggressive reduction of the tooth. In the present patient, the lateral incisors could be readily restored without aggressive reduction due to the cone shape of the teeth, allowing a minimal reduction and restoration with porcelain veneers. Hence, this was the best treatment option for the lateral incisors in our patient. Other authors [19,21] have reported a similar approach, resolving cases with porcelain materials and minimal tooth reduction, allowing the indirect restoration of laterals. The main advantage of an indirect method is that it offers absolute control over the emergence profile and considerable control over the contact area.

An anterior tooth can be restored with either direct or indirect adhesive techniques, which offer different advantages and disadvantages according to the specific clinical situation. An indirect technique involves a less conservative approach. Thus, if we had treated this patient with porcelain alone, the lateral incisor would have been prepared with a minimal reduction due to its small size, but the central incisors would have required extensive reduction. For this reason, the decision was taken to use resin composites in the central incisors, allowing restoration with minimal reduction of the enamel using a medium grit diamond bur or aluminum oxide sandblasting. Furthermore, central incisors only require minimal preparation in the interproximal area. Excellent outcomes have been reported by numerous authors who used this procedure [10,22,23].

In the present case, the predictability of the direct technique was enhanced by producing a lingual incisal silicone index to allow the creation of a stratified restoration in the mouth of the patient with the same form as a previous wax-up. This technique has been reported by numerous authors [24-26].

Different performances can be expected from the distinct materials employed in this case, which were all selected to provide long-term function and esthetics. Some studies have confirmed the long-term survival of porcelain restoration, with no change in color match or surface smoothness at 10 years [27]. Although bonded porcelain veneers deliver excellent esthetics, they have traditionally required more tooth preparation in comparison to direct composite restorations; they have shown the best overall survival [28], although their life-long survival has not been demonstrated [24]. Resin composite is less stable 
and may require more visits for maintenance. In direct composite restorations, selection of the appropriate composite materials and application techniques is critical [24]. However, composite resin can be successfully repaired, unlike porcelain $[29,30]$.

It was difficult to maintain a correct proportion of the teeth in this patient solely using a restorative approach. Most authors have generally concluded that the width of a central incisor should be roughly $80 \%$ of its length [29,31]. Various methods have been proposed to determine the appropriate width of central incisors, e. g. , by calculating oneseventh of the interpupilar distance [14], by multiplying the width of the lower central incisor by 1.62 [32], or by adding the width of a lower central incisor to one half of the width of the lower lateral incisor. Standard tooth measurements are also widely used to determine the width, ideally considered to be $8.0-8.5 \mathrm{~mm}$ for the maxillary central incisor [3,9]. The length can be determined by multiplying the width by 1.33 (maximum possible length) or 1.25 (minimum possible length). An important additional factor determining the length of a restoration is the maxillary incisor exposure at rest, reported to be a mean of 1.91 $\mathrm{mm}$ in males and $3.40 \mathrm{~mm}$ in females, with greater exposure in younger individuals and variations among ethnic groups $[3,33]$. When an ideal tooth proportion is not clinically feasible, attempts can be made to create the illusion of a more correct ratio by modifying the angles, which are clearly defined by the light reflective surface [34]. Changes in the orientation and arrangement of transition lines can increase or decrease the area of light reflection, thereby affecting the observer's perception of the size of the tooth. In the present case, this procedure was necessary due to the large width of the incisors $[35,36]$.

\section{Conclusion}

The conservative restorative approach adopted in this case satisfied the patient's demands. Dentists should fully inform patients of all possible risks, benefits and alternative options before initiating treatment. In the present patient, a substantial improvement was obtained despite the use of different materials. The natural tooth properties were replicated with minimum tooth preparation and maximum preservation of sound natural tissues.

\section{References}

1. McLaren EA, Rifkin R (2002) Macroesthetics: facial and dentofacial analysis. J Calif Dent Assoc 30: 839-846.

2. Morley J, Eubank J (2001) Macroesthetic elements of smile design. J Am Dent Assoc 132: 39-45.

3. Panossian AJ, Block MS (2010) Evaluation of the smile: facial and dental considerations. J Oral Maxillofac Surg 68: 547-554

4. Kokich VO, Kokich VG, Kiyak HA (2006) Perceptions of dental professionals and laypersons to altered dental esthetics: asymmetric and symmetric situations. Am J Orthod Dentofacial Orthop 130: 141-151.

5. Rodrigues Cde D, Magnani R, Machado MS, Oliveira OB (2009) The perception of smile attractiveness. Angle Orthod 79: 634-639.

6. Brunsvold MA (2005) Pathologic tooth migration. J Periodontol 76: 859-866.

7. Gkantidis N, Kolokitha OE, Topouzelis N (2008) Management of maxillary midline diastema with emphasis on etiology. J Clin Pediatr Dent 32: 265-272.

8. Chay SH, Ho KK (2000) Spaced dentition--open, close or redistribute? Ann R Australas Coll Dent Surg 15: 83-86.

9. Cohen M (2008) Comprehensive interdisciplinary management of patients with missing and/or abnormally proportioned teeth. Quintessence, editor. Interdisciplinary treatment planning. Principles, design, implementation. Chicago, USA 343-365.

10. Ardu S, Krejci I (2006) Biomimetic direct composite stratification technique for the restoration of anterior teeth. Quintessence Int 37: 167-174.
11. Furuse AY, Franco EJ, Mondelli $J(2008)$ Esthetic and functional restoration for an anterior open occlusal relationship with multiple diastemata: a multidisciplinary approach. J Prosthet Dent 99: 91-94.

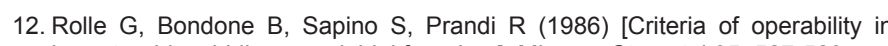
hypertrophic middle upper labial frenulum]. Minerva Stomatol 35: 527-529.

13. Sabri R, Aboujaoude N (2008) [Agenesis of the maxillary lateral incisors: orthodontic and implant approach]. Orthod Fr 79: 283-293.

14. Fradeani M (2006) Evaluation of dentolabial parameters as part of a comprehensive esthetic analysis. Eur J Esthet Dent 1: 62-69.

15. Gürel G, Bichacho N (2006) Permanent diagnostic provisional restorations for predictable results when redesigning the smile. Pract Proced Aesthet Dent 18 281-286.

16. Mizrahi B (2005) Visualization before finalization: a predictable procedure for porcelain laminates veneers. Pract Proced Aesthet Dent 17: 513-518.

17. Reshad M, Cascione D, Magne P (2008) Diagnostic mock-ups as an objective tool for predictable outcomes with porcelain laminate veneers in esthetically demanding patients: a clinical report. J Prosthet Dent 99: 333-339.

18. Nalbandian S, Millar BJ (2009) The effect of veneers on cosmetic improvement Br Dent J 207: E3.

19. Magne P, Magne M (2006) Use of additive waxup and direct intraoral mock-up for enamel preservation with porcelain laminate veneers. Eur J Esthet Dent 1 10-19.

20. Magne P (2002) Tooth preparation, impession and provisionalization Quintessence, editor. Bonded porcelain restorations in the anterior dentition: a Biomimetic approach. Chicago, USA 239-291.

21. Nazarian A (2006) Closing the gap with minimal preparation veneers. Dent Today 25: 70-71.

22. Lenhard M (2008) Closing diastemas with resin composite restorations. Eur J Esthet Dent 3: 258-268.

23. De Araujo EM Jr, Fortkamp S, Baratieri LN (2009) Closure of diastema and gingival recontouring using direct adhesive restorations: a case report. J Esthet Restor Dent 21: 229-240.

24. Fahl N Jr (2006) A polychromatic composite layering approach for solving a complex Class IV/direct veneer-diastema combination: part I. Pract Proced Aesthet Dent 18: 641-645.

25. Koczarski MJ (2005) Achieving natural aesthetics with direct resin composites: predictable clinical protocol. Pract Proced Aesthet Dent 17: 523-525.

26. Small BW (2007) Repair of central incisors on a child with diastema using a novel matrix. Gen Dent 55: 390-391.

27. Peumans M, De Munck J, Fieuws S, Lambrechts P, Vanherle G, et al. (2004) A prospective ten-year clinical trial of porcelain veneers. J Adhes Dent 6: 65-76.

28. Nový BB, Fuller CE (2008) The material science of minimally invasive esthetic restorations. Compend Contin Educ Dent 29: 338-346.

29. Duarte S Jr, Schnider P, Lorezon AP (2008) The importance of width/length ratios of maxillary anterior permanent teeth in esthetic rehabilitation. Eur $\mathrm{J}$ Esthet Dent 3: 224-234.

30. Gordan VV, Shen C, Riley J 3rd, Mjör IA (2006) Two-year clinical evaluation of repair versus replacement of composite restorations. J Esthet Restor Dent 18: 144-153.

31. Rosenstiel SF, Ward DH, Rashid RG (2000) Dentists' preferences of anterior tooth proportion--a web-based study. J Prosthodont 9: 123-136.

32. McArthur DR (1985) Determining approximate size of maxillary anterior artificia teeth when mandibular anterior teeth are present. Part I: Size relationship. J Prosthet Dent 53: 216-218.

33. Vig RG, Brundo GC (1978) The kinetics of anterior tooth display. J Prosthet Dent 39: 502-504.

34. Peyton JH (2004) Finishing and polishing techniques: direct composite resin restorations. Pract Proced Aesthet Dent 16: 293-298.

35. Fradeani M (2004) Esthetic rehabilitation in fixed prosthodontics. Quintessence editor. Esthetic rehabilitation in fixed prosthodontics. Chicago, USA.

36. Rufenacht C (2001) Principios de integración estética. 2001Quintessence, editor. Chicago, USA 63-167. 